\title{
Preattentive and cognitive effects on perceptual completion at the blind spot
}

\author{
RICK J. BROWN \\ Yerkes Regional Primate Research Center, Emory University, Atlanta, Georgia \\ and \\ JOHN B. THURMOND \\ University of Louisville, Louisville, Kentucky
}

\begin{abstract}
Our findings indicate that preattentive processes, such as the filling in of homogeneously colored areas, discrete dots, or bars across the blind spot, take into account both the color and the form that stimulate the retina around the optic disk. Perceptual completion of the "junction" of two opposite colors facing each other on opposite sides of the blind spot was resolved by simultaneous segregation of the two colors at the location of a filled-in perpendicular line that suggested a boundary separating the two colors. Orientation preference and relative salience of one color versus the other determined which color was perceptually completed in a forced-choice situation that involved perceptual completion at the intersection of a cross formed by bars of opposite colors. A 1-min exposure to these stimuli presented an ambiguous situation for perceptual completion of either color within the blind spot, and resulted in a perceptual "flip-flop" from one color to the other, much like the phenomenon that occurs in figure reversal. Instructions to speed up this reversal process led to a fivefold reduction in latency to first reversal.
\end{abstract}

The organic machinery that hums continuously below the threshold of visual consciousness seems to process optical input in a way that rapidly reduces the complexity of the world around us. These unconscious processes give us a final, comprehensive representation (at least an analogy) of the external world. Several theoretical positions currently bear on our understanding of how this is achieved. Gestaltists have insisted that we perceive meaningful wholes that are determined from the interrelations of certain visual cues (Koffka, 1935). On the other hand, recognition-by-components ( $R B C$ ) theorists have argued for the automatic recognition of so-called "geons," or visual geometric units processed independently and later reassembled (Biederman, 1987). Others have emphasized the necessity of combining the above theories in a complementary fashion in order to explain visual phenomena such as stabilized images on the retina (Pritchard, 1961).

According to these theoretical approaches, the brain plays an active rather than a passive role in the processing of visual stimuli. The well-known Kanisza triangle (Kanisza, 1976) has already become a classic example of subjective contours that the brain manufactures from incomplete figural information, synthesizing "ghosts" of geometric figures from a minimum of visual stimulation (Petry \& Meyer, 1986; Prazdny, 1985; Schumann, 1900). There is also evidence that the brain actually "patches" an incomplete visual scene. Patients affected by visual scotomas-"blind" areas of the visual field caused by

Address correspondence to J. B. Thurmond, Department of Psychology, University of Louisville, Louisville, KY 40292. migraine, retinal, or brain damage-nevertheless experience a complete picture of the world (Bender \& Teuber, 1946; Gerrits \& Timmerman, 1969; Lashley, 1941).

This filling-in process has been investigated in the region of the blind spot (a natural scotoma), an area of the retina corresponding to the optic disk where no photoreceptors exist due to the exit of retinal neurons forming the optic nerve. In 1912, Ferree and Rand argued that filling in at the blind spot occurred because the perceived space around it "shrank," that is, spatial values were believed to decrease toward the center of the blind spot. In 1929, Helson thought that the process was due to direct stimulation of the blind spot, where, he hypothesized, undiscovered photoreceptors must exist to account for perceptual completion in that area. At that time, theoretical notions regarding the filling-in phenomenon ignored the involvement of brain mechanisms.

Recent evidence (Kawabata, 1982) suggests that filling in at the blind spot is contingent upon the visual system's response to stimulation of an area of the retina directly around the optic disk, falling between $3^{\circ}$ and $5.2^{\circ}$ from the center of the blind spot; the functional size of the blind spot is $3^{\circ}$ in diameter (Menzer \& Thurmond, 1970). Filling in is also dependent upon similarity grouping, suggesting that the process of completion occurs with respect to the perceptual segregation of different stimuli (Kawabata, 1984). Apparent movement is readily seen across the blind spot (Koffka, 1924): stimulation of the retina with two points of light on opposite edges of the blind spot will evoke a perception of discrete points of light across it (Lockhead, Johnson, \& Gold, 1980). Because filling in at the blind spot cannot be predicted from 
the superimposition of local information on the retina, but seems instead to be a global process, it seems reasonable to conclude that the process governing the phenomenon occurs at higher levels in the visual system (Kawabata, 1983). However, filling in at the blind spot does not contribute to the tilt aftereffect, which indicates that filled-in contours may not be as potent in influencing higher order perception as those given by direct stimulation of the retina (Cumming \& Friend, 1980).

Since the visual system appears so reactive to presentations of incomplete stimuli that it readily synthesizes suggested forms, we investigated the system's response to stimuli that presented ambiguous possibilities for filling in. A prominent phenomenon caused by visual ambiguity is that of binocular rivalry (Kaufman, 1963; Levelt, 1967; Wolfe, 1986), a perceptual "flip-flop" occurring between two dissimilar designs presented simultaneously, but monocularly, to each eye. Fox and Check (1968) have hypothesized that an inhibitory mechanism in the visual system is responsible for this effect. Another example of visual ambiguity is the phenomenon of figure reversal, which can occur when a single design presents at least two perceptual possibilities (Brown, 1955; Flugel, 1912; Radilova, Riani, Tuccio, Radil, \& Borsellino, 1983). Figure reversal was believed to be due to the saturation of cortical neurons (Koehler \& Wallach, 1944), but it is now clear that the phenomenon has much more complex determinants. For instance, it has been demonstrated that the subjective occurrence of figure reversal depends largely on being informed of its possibility (Girgus, Rock, \& Egatz, 1977), that the frequency of the reversal process is a direct function of the ambiguity of the design (Riani, Tuccio, Borsellino, Radilova, \& Radil, 1986), and that the reversal process is subject to conscious control (Liebert \& Burk, 1985; Pelton \& Solley, 1968; Washburn \& Gillette, 1932).

Because the visual system apparently responds to both preattentive processes (i.e., the automatic synthesis of subjective contours) and the influence of higher processes (i.e., the effect of knowledge on reversal, and conscious control of the reversal process), we manipulated these factors to investigate their role in the filling-in process at the blind spot. It was hypothesized that filling in at the blind spot is governed by both an automatic process involving global visual information on the retina, and higher level processes subjected to instructions that are capable of influencing the outcome of perceptual possibilities.

\section{EXPERIMENT 1}

Experiment 1 was designed to determine if the preattentive processes governing filling in at the blind spot occur with respect to the particular hue stimulating the retina, across both primary and nonprimary colors, for homogeneous and discrete forms. Discrete forms were used to investigate global processing at the blind spot involving the interaction of two variables, form and color. The question that concerned us here was whether the stimulation of the retina with a particular hue promotes a representation of that hue within the blind spot, such that a homogeneously colored field is perceived, even though the stimuli contains a blank center positioned over the blind spot. The discrete stimulation by quasi-random dots serve to test the filling-in process with respect to its faithfulness to the form of the stimuli with which it is stimulated; thus, if the process responsible for filling in at the blind spot takes the form of the stimuli into consideration, then a field of discrete random dots of the same color as that stimulating the retina should be perceived in the blind spot, even though none is present there.

\section{Method}

Subjects. Twelve naive subjects ( 3 males, 9 females) with normal or corrected Snellen acuity of 20/20 served in the experiment. In addition, 4 subjects ( 2 males and 2 females) were run in a control condition in which all stimuli were presented outside of the blind spot. The subjects ranged in age from 18 to 35 years.

Apparatus. All stimuli were presented to the left eye, approximately $15^{\circ}$ of arc left of the center of a standard perimeter, with the subject seated at a distance of $45 \mathrm{~cm}$ from the stimuli. A chinrest with a head holder was clamped to the table supporting the perimeter to ensure rigidity of the subject's posture and to prevent movement of the head. The light illuminating the stimuli was provided by a desk lamp with a 100 -W light bulb and a fluorescent ceiling light; general illumination in the room was $965 \mathrm{~lx}$, as measured by a Litemate III photometer, Model 504 (Photo Research Inc.). The stimuli were mounted on a receptacle that slid along the perimeter so as to permit accurate superposition of each subject's blind spot with the blank center of each stimulus (see Figure 1). The center of the perimeter's vertical axis was designated by a vertical row of target points from which the subjects could pick the appropriate fixation point. A black board, cut to fit exactly over the subject's right eye, was permanently affixed to the chinrest so that viewing would occur through the left eye only.

Stimuli. All stimuli (see Figure 1) were drawn on $20 \times 15 \mathrm{~cm}$ white cards. Crayola crayons were used for coloring the stimuli so that each color could be identified by name. Eight $10 \times 10 \mathrm{~cm}$
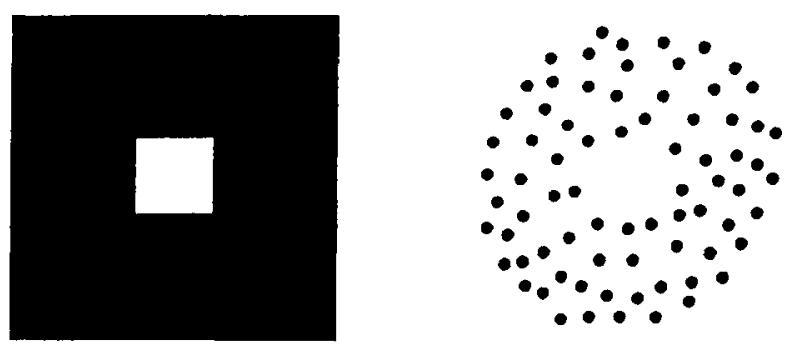

A

B

Figure 1. (A) An example of the homogeneously colored stimuli used in Experiment 1. Eight colors were used: Crayola crayons' primaries red, blue, green, and yellow; nonprimaries orchid, turquoise blue, yellow green, and orange. Each of the squares was $10 \times 10 \mathrm{~cm}\left(12.7^{\circ}\right.$ of visual angle) with a $2.5 \times 2.5 \mathrm{~cm}\left(3.2^{\circ}\right)$ blank center square. (B) An example of the discretely colored "disks." Eight stimuli were used, representing each color listed above. Each disk was composed of 98 dots, evenly distributed and positioned exactly at the same locations for all discrete stimuli. The blank center was $2.5 \mathrm{~cm}\left(3.2^{\circ}\right)$ in diameter. 
Table 1

The Munsell Matching Chips and Luminance Values for Each Stimulus Used in Experiments 1-4

\begin{tabular}{|c|c|c|c|c|c|}
\hline Color Type & Shade Used & Chip \# & Hue & $\begin{array}{c}\text { Value/ } \\
\text { Chroma }\end{array}$ & $\begin{array}{c}\text { Luminance } \\
\left(\mathrm{cd} / \mathrm{m}^{2}\right)\end{array}$ \\
\hline \multicolumn{6}{|c|}{ Experiments 1 and 2} \\
\hline Crayola & Red & 5.0 & $5.0 \mathrm{R}$ & $6 / 6$ & 123 \\
\hline Crayola & Green & 42.5 & $2.5 \mathrm{G}$ & $7 / 7$ & 111 \\
\hline Crayola & Blue & 77.5 & $7.5 \mathrm{~PB}$ & $6 / 8$ & 126 \\
\hline Crayola & Yellow & 25.0 & $5.0 \mathrm{Y}$ & $8 / 8$ & 152 \\
\hline Crayola & Purple & 95.0 & $5.0 \mathrm{RP}$ & $7 / 6$ & 140 \\
\hline Crayola & Turquoise & 57.5 & $7.5 \mathrm{BG}$ & $6 / 6$ & 108 \\
\hline Crayola & Yellow Green & 35.0 & $5.0 \mathrm{GY}$ & $8 / 8$ & 150 \\
\hline Crayola & Orange & 15.0 & $5.0 \mathrm{YR}$ & $7 / 8$ & 153 \\
\hline Background & White Paper & & & & 173 \\
\hline \multicolumn{6}{|c|}{ Experiments 3 and 4} \\
\hline Paint & Dark Red & 5.0 & $5.0 \mathrm{R}$ & $4 / 14$ & 123 \\
\hline Paint & Medium Red & 100 & $10.0 \mathrm{RP}$ & $6 / 10$ & 147 \\
\hline Paint & Light Red & 100 & $10.0 \mathrm{RP}$ & $8 / 6$ & 173 \\
\hline Paint & Dark Green & 42.5 & $2.5 \mathrm{G}$ & $5 / 8$ & 66 \\
\hline Paint & Medium Green & 42.5 & $2.5 \mathrm{G}$ & $7 / 8$ & 103 \\
\hline Paint & Light Green & 42.5 & $2.5 \mathrm{G}$ & $8 / 4$ & 155 \\
\hline Paint & Black & Neutral & & N1/black & 18 \\
\hline Paint & White & Neutral & & N9/white & 179 \\
\hline Background & White Cardboard & & & & 199 \\
\hline
\end{tabular}

Note-The numbered Munsell chips are shown with designations of hue, value (brightness), and chroma (saturation), or $\mathrm{H} \mathrm{V/C}$. The luminance values for the color of each stimulus was obtained with a Soligor Digital Spot Sensor, which detects the amount of incoming light within $1^{\circ}$ of visual angle.

squares (subtending a visual angle of $12.7^{\circ}$ ), filled homogeneously with a different color, were presented to each subject (Figure 1A). Each of these squares contained a $2.5 \times 2.5 \mathrm{~cm}\left(3.2^{\circ}\right.$ of visual angle) blank square in the center. The colors used were the four Crayola primaries red, blue, green, and yellow, and four Crayola nonprimaries, orchid, turquoise blue, yellow green, and orange. Under natural (sunlight) illumination, these colors closely matched the Munsell chips listed in Table 1 (Munsell, 1929). In addition, eight disks were constructed with a diameter of $10 \mathrm{~cm}\left(12.7^{\circ}\right)$, composed of 98 discrete dots distributed evenly and of the same colors listed above (Figure 1B). Each of these disks also had a blank center with a diameter of $2.5 \mathrm{~cm}\left(3.2^{\circ}\right)$. Thus, a total of 16 different stimuli were presented to each subject. Luminance for all stimuli (squares and disks, including the background) ranged between 185 and $380 \mathrm{~cd} / \mathrm{m}^{2}$, as measured by a Litemate III photometer. The luminance of each particular stimulus, obtained with a Soligor Digital Spot Sensor, is given in Table 1, along with the Munsell values.

Procedure. Careful tailoring of the fixation point's position for each subject was crucial because only the appropriate point would allow vertical superposition of the precise location of the subject's optic disk with the blank center of each stimulus. Using a test figure consisting of a black $2.5-\mathrm{cm}\left(3.2^{\circ}\right)$ square (see Figure 2) that corresponded exactly with the blank centers of the stimuli, proper alignment of the test figure with the blind spot was accomplished by asking the subject whether the black square disappeared completely when his/her left eye was staring at the given fixation point. The stimuli and the black square could be moved freely along the length of the perimeter, so a proper superposition was easily achieved by a trial-and-error process involving both a vertical and horizontal manipulation of eye fixation point and stimulus longitudinal position. Once the critical fixation point and the superposition of the test figure with respect to the blind spot was determined, the stimuli were presented one at a time for exactly $1 \mathrm{sec}$ to each subject. Each observer was instructed to look at the proper fixation point throughout the presentation and to report what was shown, particularly at the center of each design. The subject was probed further if answers were ambiguous or did not relate specifically to the center of the designs. No subject was informed of the blank center in the design. The order of the stimuli was completely randomized before presentation to each subject.

\section{Results}

The homogeneously colored squares and discrete dot patterns (Figure 1) presented at the blind spot were readily filled in by all the subjects for each of the 16 stimuli. As a control, the 16 stimuli were presented at the same eccentricity as, but outside of, the blind spot. In no instance did completion occur in this condition. At the blind spot, the homogeneous squares were perceived as regular, continuously colored figures, even at the center; the

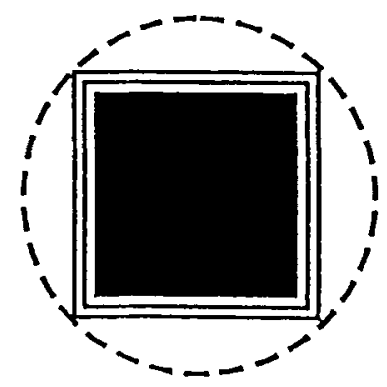

Figure 2. The test figure used for determining the precise location of the blind spot. The dotted line is a schematic representation of the area of the blind spot as it would fall over the black square. The blind spot was judged to fall exactly over the square when the square was reported by the subject to disappear completely, that is, when the white background of the card on which the black test figure was drawn would fill in across the blind spot. The thin outline around the square was used to determine whether the subject's blind spot was too small to cover the square completely, in which case the subject would report an outline with a blank center. 
discrete dot patterns were perceived as a collection of dots, all of the same color, with the same distribution and density in the center. The presence of these illusory dots was reported "matter-of-factly," as the subjects were unaware that the stimuli were actually blank in the center. The subjects, when asked, denied that they noticed anything unusual about the stimuli. This suggests that the dots appeared to be distributed across the blind spot with no significant quantitative or qualitative perceptual differences with the surrounding stimulated area of the retina. This also implies that the dots must have occupied precise positions in the blind spot, as was the case when one of the authors tested himself. At the end of the experiment, each subject expressed considerable surprise upon discovering that the designs were actually blank in the center.

\section{EXPERIMENT 2}

In Experiment 2, we tested the hypothesis that a preattentive factor, such as the presentation of a contour boundary, leads to a reduction in ambiguity regarding filling in. Here, we wanted to demonstrate that when two bars of different colors on opposite sides of the blind spot are competing for filling in, a line placed perpendicularly to the colored bars (see Figure 3) facilitates a perceptual segregation of hue in the blind spot. Hence, rather than coming to an arbitrary stopping place or mixing together, the colors should meet close to the filled-in perpendicular line.

\section{Method}

Subjects. Ten naive subjects, 4 males and 6 females, participated; they ranged in age from 20 to 30 years. All had normal or corrected 20/20 Snellen acuity.

Apparatus. The standard perimeter and accessories were the same as those used in Experiment 1.

Stimuli. The stimuli were the same overall size as those used in Experiment 1; diagrams are shown in Figure 3. Four $1.5 \mathrm{~cm}$ $\left(1.9^{\circ}\right)$ wide $\times 10 \mathrm{~cm}\left(12.7^{\circ}\right)$ long bars were drawn vertically, the top halves colored with red crayon and the bottom halves with a complementary green (see Table 1). The center was kept blank so that the two halves of each bar were $1.9 \mathrm{~cm}\left(2.4^{\circ}\right)$ apart. Two halves of one bar with no added line served as one stimulus (Figure $3 \mathrm{~A}$ ); another bar had a black line drawn perpendicularly to it, as if it would cross its very center, although the center was kept blank for $1.9 \mathrm{~cm}\left(2.4^{\circ}\right.$ ) across the bar (Figure 3B). Another bar had the same black divided line drawn perpendicularly to it and $0.3 \mathrm{~cm}$ below the bottom edge of the top bar (Figure 3C); the fourth bar also had a black divided line at right angles to it, but this line was $0.3 \mathrm{~cm}$ above the top edge of the bottom bar (Figure 3D). Luminance values for the above stimuli (bars and background) were between 200 and $350 \mathrm{~cd} / \mathrm{m}^{2}$, as recorded by the Litemate III photometer. Luminance values for each particular stimulus are given in Table 1 . The order of presentation of the four bars was counterbalanced across subjects.

Procedure. The procedure was the same as that used in Experiment 1 , except that each subject was instructed to "Tell me what you see, especially in the middle." The entire response was recorded and, if ambiguous, the subject was probed further to evoke a specific answer; that is, "Do the colors meet or not? [if yes] Where?"'
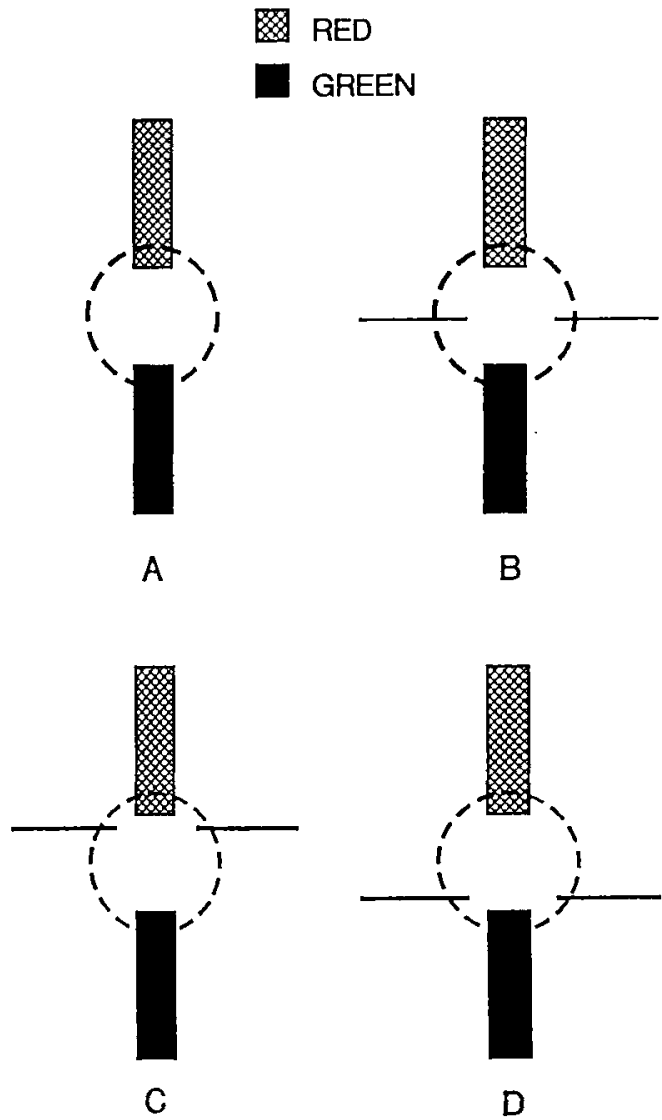

Figure 3. The stimuli used in Experiment 2 for determining whether or not the blind spot uses boundary information (the incomplete horizontal line) for filling in two opposite colors. Dotted lines represent the location of the blind spot. (A) Two half-bars, 1.5 $\left(1.9^{\circ}\right) \times 10 \mathrm{~cm}\left(12.7^{\circ}\right)$, the top one red, the bottom one green, would be filled in by the blind spot by reaching a compromise between the two colors, that is, by segregating them somewhere in the blank area, or by selecting a single color. The same stimuli were presented with a line drawn horizontally to the half-bars in the middle of the blank area (B), $.3 \mathrm{~cm}$ below the top bar (C), and $.3 \mathrm{~cm}$ above the bottom bar (D).

\section{Results}

Of the 10 subjects in the "no line" condition (Figure $3 \mathrm{~A}$ ), 5 could not say where the colors met. A typical response was: "It's funny; I can tell they meet but I can't say where!" The other half of the subjects reported that the colors met "somewhere in the middle." For the bar comprising the perpendicular line condition (Figures 3B-3D), all the subjects perceived the perpendicular line as being complete across their blind spot; 9 of the 10 subjects (including those in the no line condition who reported that they could not tell where the colors met) reported that the colors definitely met. If the line had no effect, half of the subjects ( 5 out of 10 ) would be expected to report that the colors met. Thus, the frequency of subjects reporting that the colors met depended on whether the line 
was present or not. A chi-square analysis applied to these frequency data yielded significant differences between these conditions $\left[\chi^{2}(1)=6.40, p<.02\right]$.

\section{EXPERIMENT 3}

The purpose of Experiment 3 was to compare the effects of one color with another on filling in at the blind spot, with respect to saturation, brightness, and contrast with the background. Given that two orientations (vertical and horizontal) competed for filling in, it was hypothesized that the more salient color would have a tendency to fill in at the blind spot regardless of orientation, but that an initial orientation preference would still bias filling in toward that preferred orientation.

\section{Method}

Subjects. Sixteen naive subjects ( 7 males and 9 females) were used as subjects. The 4 subjects who were run as a control group in Experiment 1 served in a control condition in which all stimuli were presented outside of the blind spot. All ranged in age between 20 and 40 years and had normal or corrected $20 / 20$ Snellen acuity.

Apparatus. The perimeter and accessories were the same as those used in Experiment 1.

Stimuli. Twenty crosses (see example in Figure 4), with a blank $1.9 \times 1.9 \mathrm{~cm}\left(2.4^{\circ}\right)$ square in each center, were mounted successively on the perimeter. All the crosses were composed of bars that were $1.3 \mathrm{~cm}\left(1.7^{\circ}\right)$ wide $\times 10 \mathrm{~cm}\left(12.7^{\circ}\right)$ long, the blank center accounting for $1.9 \mathrm{~cm}$ of the length. The colors for these stimuli were red, green, black, and white from Tempesta poster paint, a liquid paint that can be readily mixed to provide gradients of desaturation by adding white to a given color. Eighteen crosses were chromatic, being colored red and green, and two were achromatic, that is, black and a light shade of gray obtained by mixing 14 parts white to one part black. The black color matched the Munsell chip designated as black (N1), and the light gray matched the white chip (N9; N10 is actually white). Matching Munsell values for each of the paints used are shown in Table 1 .

The crosses were composed of bars of opposing colors: red versus green and black versus light gray. For the chromatic crosses, salience (or color) values of 1,2 , and 3 were arbitrarily assigned to

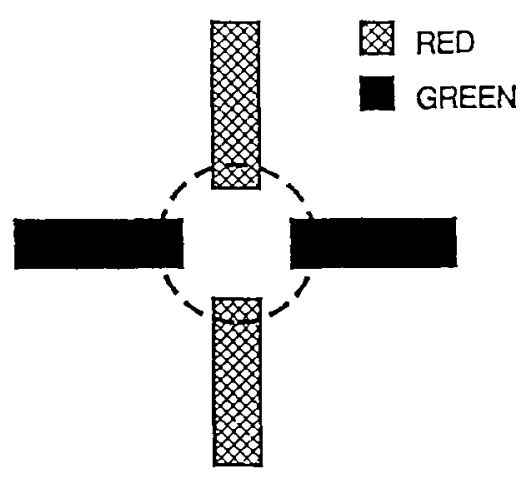

Figure 4. An example of the crosses used in Experiments 3 and 4. Here, the vertical bar, $1.3 \mathrm{~cm}\left(1.7^{\circ}\right) \times 10 \mathrm{~cm}\left(12.7^{\circ}\right)$, represents the color red and the horizontal bar of the same dimensions represents the color green. These two orientations for color, however, were reversed for the counterbalanced stimuli. Two of the crosses were achromatic (black and light gray). The dotted line represents the blind spot overlapping the blank area of the stimuli. the real ratios, derived by adding 0,2 , and 14 parts white to 1 part of the given color, respectively. Since only relative salience was relevant to this experiment, it did not matter what the absolute salience values were for a given color, thereby justifying an arbitrary choice of salience values signifying an actual increase in the physical ratios. Thus, as the salience of the vertical bar increased relative to the horizontal bar, whether or not the color of the vertical bar was red or green (the horizontal color was always opposite, i.e., complementary, to the vertical color), the ratio of the two salience values (vertical:horizontal) increased. In the extreme cases, a ratio of 1:3 red indicates that, for the cross on this particular stimulus card, the vertical red had a salience value of 1 , whereas the horizontal green paired with it had a salience value of 3 . This also means that red had a relative salience of 0.33 relative to the green (this decimal value was actually used to describe relative salience in Figure 5). Conversely, a ratio of 3:1 for red indicates that the vertical red had a salience of 3 , whereas the horizontal green had a salience value of 1 , yielding a decimal value of 3.00 in favor of the vertical bar (red, in this case). For both the red vertical bar (green horizontal bar) and the green vertical bar (red horizontal bar) the ratios of salience were increased systematically to give the following decimal values: $0.33(1: 3), 0.50(1: 2), 0.67(2: 3), 1.00(1: 1)$, $1.00(2: 2), 1.00(3: 3), 1.50(3: 2), 2.00(2: 1)$, and $3.00(3: 1)$. The nine crosses with red kept vertical and green kept horizontal were counterbalanced by the complementary set of the reversed orientation (green vertical and red horizontal). For the achromatic crosses, only two levels of salience were used: black and a very light gray, the latter being just dark enough to provide a contrast with the white of the card upon which it was applied. The vertical black/horizontal gray cross was counterbalanced by the vertical gray/horizontal black cross. The order of all 20 crosses (chromatic and achromatic) was randomized before presentation to each subject. Luminance values (crosses and background), as measured by the Litemate III photometer, were between 90 and $320 \mathrm{~cd} / \mathrm{m}^{2}$ for the chromatic stimuli, and approximately $60 \mathrm{~cd} / \mathrm{m}^{2}$ for black and $400 \mathrm{~cd} / \mathrm{m}^{2}$ for gray. The luminance values for each particular stimulus, obtained with the Soligor Digital Spot Sensor, are given in Table 1.

Procedure. Each subject was categorized as being either "vertical" or "horizontal" by pretesting their preference for a particular orientation. To determine this, the investigator asked the subject to report the orientation of the bar that he/she filled in when presented with the red/green and green/red (color counterbalanced) cross, both with the relative salience $1.00(3: 3)$ in his/her blind spot. The procedure was exactly the same as that used in Experiment 1, except that each subject was instructed as follows: "Tell me which bar you see to be 'on top' of the other by naming the color associated with that bar, that is, if the red bar is on top, or crossing the green, say 'red'; if instead the green is on top, say 'green.' "' The usage of the term "on top" was justified from the standpoint that naive subjects who served in Experiment 1 interpreted the filling in of one bar as the corresponding color lying "above," or crossing the other, thereby completely "hiding" the bar "underneath."

\section{Results}

As in Experiment 1, none of the crosses presented outside of the blind spot (at the same eccentricity) were filled in.

Saturation. Figure 5 shows the relationship between relative salience of a color and the number of subjects perceptually completing the blind spot with that color. The percentage of subjects filling in the red vertical bars in the red/green crosses and the green vertical bars in the green/red crosses are indicated with respect to each vertical bar's salience relative to the horizontal bar. It is apparent that the number of subjects filling in the vertical 


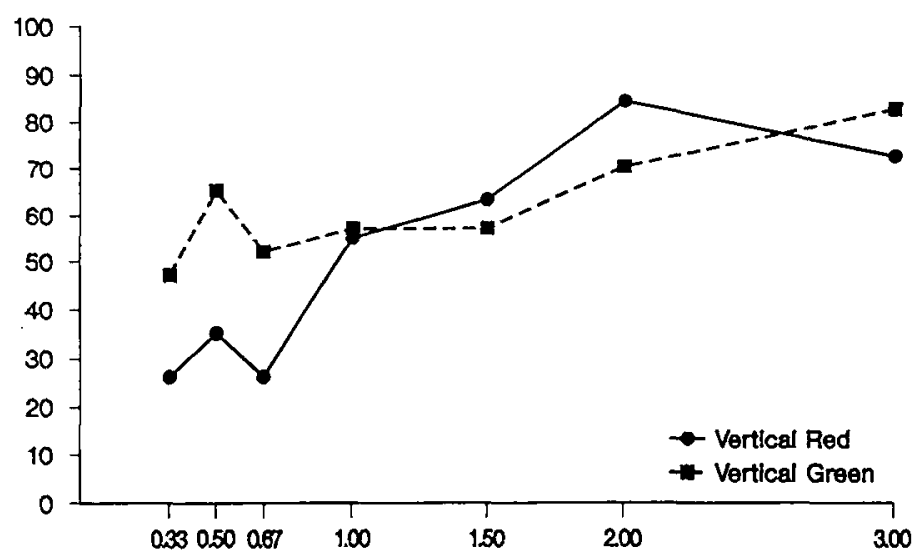

Relative Saturation (Vertical vs. Horizontal Hue)

Figure 5. Percentage of subjects filling in a particular color as a function of that color's salience relative to the complementary color in Experiment 3 . The midde relative salience of 1.00 is actually the mean value obtained by averaging three ratios $(1: 1,2: 2$, and $3: 3)$. The vertical orientation was chosen arbitrarily to represent each color's relative salience for the sake of simplicity and to avoid being redundant; the horizontal orientation was complementary (red or green) to the vertical.

bar increases as the relative salience of that bar increases with respect to the horizontal bar, regardless of the color (red or green). Note that the effect of increasing relative salience of the horizontal bar with respect to the vertical bar is easily observed from the data of Figure 5. For example, approximately $25 \%$ of the subjects perceived the red vertical bar to be filled in when the relative salience of vertical red with respect to horizontal green was 0.33 , so the complementary percentage, or $75 \%$, perceived the horizontal green, instead, to be filled in.

If one follows the percentage data from left to right in Figure 5 indicating subjects filling in the horizontal bar, it can be seen that this percentage decreases as the relative salience of the horizontal bar decreases (from left to right, absolute salience of vertical bar increases, while the absolute salience of the horizontal bar decreases). It can also be seen that at the opposite end of the salience scale, approximately $75 \%$ of the subjects perceived the red vertical bar to be filled in, whereas $25 \%$ perceived the green horizontal bar to be filled in. Because the two lines (for vertical red and vertical green) are roughly linear with similar slopes, it seems safe to conclude that the results regarding the effects of salience are essentially the same for both the horizontal and vertical orientations.

Orientation. For the chromatic stimuli, the percentage of bars that each subject perceived to be "on top" (number of bars filling in) was always greater in favor of his/her initial orientation preference, regardless of whether the red bar was vertical (green horizontal) or vice versa (see Table 2). The percentage of bars perceived to be filled in according to the initial preference of the subjects turned out to be significant across color. The mean percentage of filling in with respect to initial orientation preference was $83.95 \%$ for the vertical subjects and $74.61 \%$ for the horizontal subjects. A single sample $t$ test (one-tailed) was performed on the data for vertical and horizontal subjects separately, by comparing the mean percentage of occurrence of one orientation versus the predicted frequency of occurrence in accordance with the null hypothesis $(50 \%)$. The effect of vertical preference was found to be significant $[t(8)=5.80, p<.0005]$, as was the effect of horizontal preference $[t(6)=3.23, p<.01]$.

The results (Table 2 ) for the black and gray crosses suggest an interaction effect between salience and orientation when the difference in salience between two achromatic colors is large. The fact that black was filled in more frequently than gray, regardless of the orientation, suggests that colors providing higher contrasts fill in better. In addition, since the filling in of gray occurred much more frequently for the vertical than for the horizontal orientation, it is tempting to conclude that the vertical orientation is preferred. This conclusion receives further support from the data obtained for the filling in of black (88.89\% for vertical compared with $\mathbf{8 5 . 7 1 \%}$ for horizontal) and the higher percentage values obtained for the vertical filling in of chromatic stimuli by the subjects with

Table 2

Mean Percentages of Filled-in Bars Corresponding to Initial Orientation Preference in Experiment 3

\begin{tabular}{cccccc}
\hline & \multicolumn{4}{c}{ Color } \\
\cline { 2 - 3 } $\begin{array}{c}\text { Orientation Preference } \\
\text { of Subject }\end{array}$ & \multicolumn{2}{c}{ Chromatic } & & \multicolumn{2}{c}{ Achromatic } \\
\cline { 6 - 7 } \cline { 5 - 6 } & Red & Green & & Black & Gray \\
\hline Vertical & 81.48 & 86.42 & & 88.89 & 55.56 \\
Horizontal & 66.67 & 82.54 & & 85.71 & 28.57 \\
\hline
\end{tabular}


a vertical orientation preference versus the horizontal filling in of chromatic stimuli by the subjects with a horizontal preference. An independent groups $t$ test (two-tailed) applied to the overall difference in mean percentage (based on both chromatic and achromatic stimuli) between the subjects with a vertical preference $(81.5 \%)$ and those with a horizontal preference $(69.8 \%)$ was significant $[t(14)=$ $3.42, p<.01]$.

Color. Table 2 indicates a preference for the color green across the chromatic stimuli and orientation. Figure 5, which describes results on salience, supports this tendency, as more of the subjects filled in green on the average. Across all chromatic stimuli, regardless of orientation preference (horizontal or vertical), the subjects filled in $54 \%$ of the green bars and thus $46 \%$ of the red bars, as red and green bars were always paired. A correlated groups $t$ test (two-tailed), comparing these two mean values separately for the two subgroups of subjects, yielded a statistically significant difference for those with a horizontal orientation preference $[t(7)=2.67, p<.05]$ as well as for those with a vertical orientation preference $[t(7)=4.20, p<.01]$.

\section{EXPERIMENT 4}

In Experiment 4, we tested the hypothesis that perceptual completion at the blind spot is subject to reversal. We also tested the hypothesis that awareness of the possibility of reversal would exert a direct influence on completion reversal. Therefore, Experiment 4 was designed to determine (1) if reversal occurs regarding the filling in of the vertical versus the horizontal bar, and (2) if an awareness of the possibility of reversal has any influence on the reversal process.

\section{Method}

Subjects. Twelve trained observers participated in Experiment 4 They were given practice on filling in with the crosses but were naive about the possibility of reversing the filling-in process. Five were males and 7 were females, ranging in age from 18 to 30 years.

Apparatus. The apparatus was exactly the same as that used in Experiment 1.

Stimuli. Four cards were selected from the set of crosses used in Experiment 3: the chromatic crosses (red vertical:green horizontal) with relative salience ratios of $1.00(3: 3), 1.00(1: 1)$, and 3.00 (3:1), and one achromatic cross (black vertical:gray horizontal). The four cards are referred to in Table 3 as A, B, C, and D, respectively.

Procedure. The subjects were divided into two groups of 6 each with an approximately equal number of males and females. The first group (naive group) was randomly assigned to the condition under which the reversals were to be performed first, without any previous experience in reversing the filled-in patterns (passive condition). These subjects were instructed to observe each cross (in their blind spot) for a full minute, and to report any change whatsoever to the investigator. Each subject was told that fading of the design or the color was normal under relatively long visual exposure, and that a change of that nature was not to be reported. Responses regarding the reversal latencies were timed with a stopwatch (in seconds). Latency was defined as the period between first exposure to the stimuli and first reversal, signaled by a response such as "the horizontal bar is no longer on top; now the vertical is," or similar statements implying that a reversal of filling in of color was taking place. The order of presentation of the stimuli was completely randomized. After the subjects had responded to all four stimuli, they were presented with the same crosses in the initial order, but with instructions to attempt to "bring the bar perceived to be 'under' 'on top' instead," that is, to reverse the filling in to the other color, and to keep reversing as fast as possible (instructed condition). Exactly $1 \mathrm{~min}$ was allowed for the observation of each cross (in order to keep observation time constant across the passive and instructed conditions), during which time the subject gave a continuous report of reversal, for example, "red is on top. . green... red..." and so forth. Each response that corresponded to a reversal was timed, again with a stopwatch, by simultaneously going down a list of values between 1 and $60 \mathrm{sec}$ and putting a check mark beside the appropriate value at the time of the response. Only the latencies for first reversal were used in the computation of the data, however, since repetition of reversals (suggested by the experimenter in the instructed condition) during the $1 \mathrm{~min}$ of observation was executed by the subjects only for the sake of acquiring experience with manipulation of the reversal process.

The second group of subjects (experienced group) was given the same basic instructions as the naive group, but the order of the task was reversed. Each subject was instructed to reverse the filling in of the colors voluntarily for a full minute (instructed condition), and was then asked to passively observe the stimulus for another full minute (passive condition). Appropriate alterations of the instructions were made to accommodate the new order of presentation; once a subject was aware of the possibility of initiating reversals, he/she was no longer a naive subject, so that, instead of "reporting any change in the stimuli," each subject was asked to observe the stimuli with a passive attitude, and to report reversals as they occurred. Timing for the reversals was accomplished in the same manner as for the naive group.

\section{Results}

Table 3 shows the mean latencies obtained for each of the four stimulus cards (A, B, C, and D) under each condition. It can be seen from Table 3 that, compared with the passive condition for both groups, in which reversals occurred without any instructions on the part of the experimenter, the instructions to initiate reversals (instructed condition) resulted in a marked reduction in the mean latency to first reversal for the four stimuli. The mean latency for first reversals across the four stimuli computed for the passive conditions over both groups was $39.25 \mathrm{sec}$; however, the mean latency for the instructed conditions over both groups was $9.6 \mathrm{sec}$. A $t$ test for correlated groups (two-tailed) comparing these latency values for passive and suggested reversals was found to be statisti-

Table 3

Mean Latencies (in Seconds) of Passive and Instructed Reversals Reversals in Naive and Experienced Subjects for Four Different Crosses (Stimulus Cards) in Experiment 4

\begin{tabular}{|c|c|c|c|c|c|}
\hline \multirow[b]{2}{*}{ Condition } & \multicolumn{4}{|c|}{ Crosses } & \multirow{2}{*}{$\begin{array}{l}\text { Grand } \\
\text { Mean }\end{array}$} \\
\hline & A & B & $\mathbf{C}$ & $\mathrm{D}$ & \\
\hline \multicolumn{6}{|c|}{ Naive Subjects } \\
\hline $\begin{array}{l}\text { Passive } \\
\text { Instructed }\end{array}$ & $\begin{array}{l}58.5 \\
11.0\end{array}$ & $\begin{array}{r}37.7 \\
8.8\end{array}$ & $\begin{array}{l}43.4 \\
10.0\end{array}$ & $\begin{array}{r}57.5 \\
6.8\end{array}$ & $\begin{array}{r}49.3 \\
9.7\end{array}$ \\
\hline \multicolumn{6}{|c|}{ Experienced Subjects } \\
\hline $\begin{array}{l}\text { Passive } \\
\text { Instructed }\end{array}$ & $\begin{array}{r}23.2 \\
6.3 \\
\end{array}$ & $\begin{array}{l}26.2 \\
13.1\end{array}$ & $\begin{array}{r}40.2 \\
6.5\end{array}$ & $\begin{array}{l}27.3 \\
12.7\end{array}$ & $\begin{array}{r}29.2 \\
9.5\end{array}$ \\
\hline
\end{tabular}


cally significant $[t(11)=6.24, p<.001]$. It is apparent from the data shown in Table 3 that the latencies to first reversal under the instructed condition were approximately the same for the naive and experienced subjects (mean values across the four stimulus cards were 9.7 and $9.5 \mathrm{sec}$, respectively). However, an examination of Table 3 reveals a marked reduction in the passive reversal latencies when the subjects had prior experience in attempting to influence the reversal process; the effect is statistically significant, as determined by a $t$ test (twotailed) for independent groups $[t(10)=5.58, p<.001]$.

It is worth noting that when the passive condition was tested first (naive group), in 15 out of 24 trials ( 4 stimuli $\times 6$ subjects) the subjects never reported passive reversals of the crosses. However, when the passive condition was tested after the instructed condition (experienced group), only on 5 out of 24 trials did the subjects not report reversals. A value of $60 \mathrm{sec}$ was assigned as a latency value to these "no reversal" trials (due to the 1-min time limit) in order to represent latency as a number for the sake of computation. Had latencies for the no-reversal periods not been held artificially to $60 \mathrm{sec}$, the effect of the order of presentation between the naive and experienced groups would have been stronger.

\section{GENERAL DISCUSSION}

Variations in fixation due to eye movements may be expected to affect the experimental results obtained in the present study. However, Kawabata (1984) has discussed at length the possible effects of eye movements, so the discussion will not be repeated here. He concluded that perceptual completion at the blind spot was not significantly affected by eye movements.

Several factors influence filling in at the blind spot. One preattentive process is the seemingly instant completion of homogeneous color as well as the filling in of discrete dot patterns, across both primary and nonprimary hues. Our findings indicate that this process is not simple, but rather is subject to global interactions of visual information on the retina. A boundary contour, such as a line drawn horizontally with respect to the blind spot, is perceived as complete across the blind spot, forcing the vertical colors to fill in "around it," presumably because the line is a much more prominent (darker) feature. The line also facilitates the resolution of ambiguity that is due to two complementary colors competing for filling in vertically in the blind spot. This competition between the two colors was unresolved in the case where no line was provided; that is, in the "no line condition," half of the subjects reported that the colors met in the middle, which is what is expected by chance, or guessing. The remaining half of the subjects were unable to come to a decision and could not say where the colors met. The issue, however, is whether the horizontal boundary contour truly prompted a global process that ultimately resulted in the perception of the colors as clearly meeting at, or very near, the line. Alternatively, perception of the colors as meeting at a specific location within the blind spot could have been due to a rationalization or a guess caused by independent processing of the horizontal line. In other words, the observer may have decided that, because there was a line crossing between the two half-bars, the opposite colors probably met at that line.

Although a cognitive influence on the perception of the colors meeting at a precise location cannot be ruled out, it should be noted that the subjects were particularly clear and convincing in their verbal report that the colors met at or near the line regardless of the line's position with respect to the vertical axis. Results obtained by Kawabata (1984) can be interpreted to indicate that perceptual completion of a horizontal pattern across the blind spot in competition with a pattern forming a vertical blank stripe occurred only if the time lag between presentation of the two patterns exceeded $50 \mathrm{msec}$. Further examination of these prior findings reveals that maximum perceptual completion of a horizontal line across the blind spot occurs in approximately $120 \mathrm{msec}$. The stimuli in the present study were presented to the subjects for $1,000 \mathrm{msec}$, so ample time was available for perceptual completion of the line across the blind spot, although little time was available for cognitive processing, unless it occurred during the poststimulation period.

Other preattentive factors contributing to global processing of filling in at the blind spot are orientation preference and relative "salience." An initial orientation preference biases the visual system toward perceptual completion with respect to the preferred orientation in a forced-choice situation. The ultimate factor "tipping" the completion phenomenon in an ambiguous stimulus situation toward one of the two visual possibilities may be due to individual predispositions in general. Given the stability of the orientation preference (vertical vs. horizontal) across color, it is unlikely that filling in of a particular orientation is due to a process higher than the preattentive level. Rather, it seems evident that the consistency of perceptual completion with respect to a particular orientation indicates that an established propensity influences the filling-in phenomenon. It is interesting to note that the present results revealed a preference for the vertical orientation versus the horizontal orientation. In light of the evidence obtained in prior studies indicating a general preference for vertical and horizontal orientations as opposed to oblique (Mansfield, 1974; Orban, Vandenbusshe, \& Vogels, 1984), it would be especially instructive to investigate this preference relative to the filling-in phenomenon.

Our results indicate that a forced-choice situation created by a difference in color salience between two stimuli leads to filling in in favor of the more salient color. This also no doubt accounts for the relative dominance of the color green over red (Figure 5), because the green paint provided more contrast (see Table 1) than the bars colored with red. Apparently, this completion phenomenon is distinct from other illusions, such as transparency effects. Every subject reported completely filled-in pat- 
terns, such that the bar that was subjected to completion seemed to be cutting through or "hiding" the perpendicular pattern. In other words, completion of one pattern left no room for completion of the other. This was also obvious to the authors who tested themselves.

We propose that filling in at the blind spot is likely to occur in Area 17 of the occipital cortex upon activation of neurons that receive afferents from the ipsilateral eye. LeVay, Connolly, Houde, and Van Essen (1985) have used transneuronal autoradiography to demonstrate that the cortical locus topographically identified as the blind spot is completely filled in by fibers serving the ipsilateral eye, which represent the optic disk (blind spot) in the contralateral eye. Thus, the excitatory postsynaptic potentials (EPSPs) of cortical cells responding to the retinal region just outside the optic disk could, in principle, spread inwardly to the monocular cells serving the other eye. This would especially be the case if the fellow eye was occluded, hence providing no competition in the activation of the "cortical blind spot." Under monocular stimulation of the surrounding area of the blind spot, stimuli with the highest contrasts are more likely to provide the strongest inputs inwardly to neurons serving the other eye than are stimuli with lower contrasts. Therefore, a stimulus having a higher contrast is more likely to fill in perceptually.

Two mechanisms may be influencing the EPSP's spread to the cortical blind spot. Neuronal fatigue may be reducing the strength of inputs from one set of neurons responding to a particular contrast, even if the latter is high relative to its alternative. In this case, the second set of neurons responding to the alternative contrast may have enough relative strength to compete with the former. Influence from higher areas may feed back onto the area immediately surrounding the cortical blind spot and change the weighted inputs from the alternative sets of neurons to the neurons serving the cortical blind spot. Extensive feedback to Area 17 from higher cortical areas is in fact present in the macaque monkey (Van Essen, 1985 ), a primate species that has a visual system very similar to that of humans (Boothe, Dobson, \& Teller, 1985). Verification of these notions could be obtained by direct single-cell recordings inside the cortical blind spot to examine the response to monocular stimulation of the area immediately surrounding the optic disk, and psychophysical investigations of interocular transfer from the eye serving the interior of the cortical blind spot to the eye in which the filling in is observed.

If the visual system is exposed to a forced-choice situation, such as filling in either of two colors (red or green), or two stimuli differing in contrast (black and gray), then filling in occurs according to global, preattentive processes, as described above. However, within approximately $1 \mathrm{~min}$, a reversal of filling in can take place, bringing forth the alternate perceptual possibility. The reversals observed in the present study seem remarkably similar to those obtained under conditions of binocular rivalry (Wolfe, 1986) or the reversal of figures when single designs present perceptual alternatives (Riani et al., 1986). Pritchard (1961) observed that similar perceptual alternatives occurred during time periods when images on the retina were stabilized. In the case of monocularly presented stimuli in the present study, it seems likely that the reversal of perceptual alternatives is related to the operation of figure-ground processes in the visual system. According to Gestalt principles (Koffka, 1935), the stimuli used in Experiment 4 offered no clear cues regarding a figure-ground relationship, hence the ambiguity leading to reversals.

Our findings indicate that filling-in reversal at the blind spot is automatic, but it occurs more quickly when instructions are given to facilitate its occurrence. It is clear from the results of Experiment 4 that a marked decrease in latency to reversal occurred for the instructed condition, which was consistent across stimuli and remarkably stable regardless of the order of presentation. Based on the data obtained on figure reversal by Girgus et al. (1977), it is probable that this facilitation was in fact due to the acquired knowledge that reversal is possible. One could argue, however, that the difference in reversal latency observed between the two conditions was perhaps due to an inhibition of reversal during the passive condition that was released during the instructed condition. Such inhibition could arise from the subjects' attempt to maintain fixation on the target. This does not imply direct conscious control, but it does suggest that the preattentive process of filling in at the blind spot is subject to cognitive influence, that is, a different attitude, state, or predisposition toward the stimuli, be it in the form of directly facilitating reversals or disinhibiting a process responsible for "blocking" reversals.

In summary, our evidence suggests that a stimulus configuration surrounding the blind spot will be filled in, true to form, whether the surround is a homogeneous color or a repetitive pattern. Moreover, filling in at the blind spot is apparently unique, because it was found that completion did not occur outside of the blind spot. At the blind spot, the figures that were formed on the basis of similar color or salience in the present study were affected by global processes, such as the presence of a horizontal line that altered the perceived relative sizes of vertical rectangles filled in at the blind spot, thereby resolving a perceptual ambiguity. We also found that it was possible for the subjects to reverse figures that were competing for completion across the blind spot, much like the occurrence of perceptual alternatives associated with figure reversal. The striking resemblance between filling-in reversal at the blind spot and figure reversal makes it tempting to assign a common mechanism to both processes. Perhaps this mechanism is related to processes in the visual system that are responsible for separating figure (in front or on top) from ground (in back or underneath), the effects of which are seen here operating under ambiguous figure-ground stimulus conditions. 


\section{REFERENCES}

Bender, M. B., \& Teuber, H. C. (1946). Phenomena of fluctuation, extinction and completion in visual perception. American Medical Association Archives of Neurology \& Psychiatry, 55, 267-268.

BIEDERMAN, I. (1987). Recognition-by-component: A theory of human image understanding. Psychological Review, 94, 115-147.

Boothe, R. G., Dobson, V., \& Teller, D. Y. (1985). Postnatal development of vision in human and nonhuman primates. Annual Review of Neuroscience, 8, 495-545.

Brown, K. T. (1955). Rate of apparent change in a dynamic ambiguous figure as a function of observation time. American Journal of Psychology, 68, 358-371.

Cumming, G., \& Friend, H. (1980). Perception at the blind spot and tilt aftereffect. Perception, 9, 233-238.

FERrEe, C. E., \& RAND, G. (1912). The spatial values of the visual field immediately surrounding the blind spot and the question of associatively filling-in of the blind spot. American Joumal of Psychology, 29, 398-417.

Flugel, J. C. (1912). The influence of attention in illusions of reversible perspective. British Journal of Psychology, 5, 357-416.

Fox, R., \& CHeCK, R. (1968). Detection of motion during binocular rivalry suppression. Joumal of Experimental Psychology, 78, 388-395.

Gerrits, H. J. M., \& Timmerman, J. G. M. E. N. (1969). The fillingin process in patients with retinal scotomata. Vision Research, 9, 439-442.

Girgus, J. J., Rock, I., \& Egatz, R. (1977). The effect of knowledge of reversibility on the reversibility of ambiguous figures. Perception \& Psychophysics, 22, 550-556.

Helson, H. (1929). The effect of direct stimulation of the blind spot. American Journal of Psychology, 41, 345-397.

Kanisza, G. (1976). Subjective contours. Scientific American, 234, 48-52.

KaUFMAN, L. (1963). On the spread of suppression and binocular rivalry. Vision Research, 3, 401-415.

KaWABATA, N. (1982). Visual information processing at the blind spot. Perceptual \& Motor Skills, 55, 95-104.

KaWABATA, N. (1983). Global interactions in perceptual completion at the blind. Vision Research, 23, 275-279.

Kawabata, N. (1984). Perception at the blind spot and similarity grouping. Perception \& Psychophysics, 36, 151-158.

Koemler, W., \& Wallach, H. (1944). Figural aftereffects: An investigation of visual processes. Proceedings of the American Philosophical Society, 88, 269-357.

KoFFKA, K. (1924). The perception of movement in the region of the blind spot. British Journal of Psychology, 14, 269-273.

Koffik, K. (1935). Principles of Gestalt psychology. New York: Harcourt Brace Jovanovich.

LASHLEY, K. S. (1941). Patterns of cerebral integration indicated by the scotomas of migraine. Archives of Neurology \& Psychiatry, Chicago, 46, 331-339.
LeVay, S., Connolly, M., Houde, J., \& Van Essen, D. C. (1985). The complete pattern of ocular dominance stripes in the striate cortex and visual field of the macaque monkey. Joumal of Neuroscience, $5,486-501$.

LEVELT, W. J. M. (1967). Note on the distribution of dominance times in binocular rivalry. British Joumal of Psychology, 58, 143-145.

LIEBERT, M., \& BURK, B. (1985). Voluntary control of reversible figures. Perceptual \& Motor Skills, 61, 1307-1310.

Lockhead, G. R., Johnson, R. C., \& Gold, F. M. (1980). Saltation through the blind spot. Perception \& Psychophysics, 27, 545-549.

MANSFIELD, R. (1974). Neural basis of orientation perception in primate vision. Science, 186, 1133-1135.

Menzer, G. W., \& Thurmond, J. B. (1970). Form identification in peripheral vision. Perception \& Psychophysics, 8, 205-209.

Munsell, A. H. (1929). Munsell book of color. Baltimore: Munsell Color Company, Inc.

Orban, G. A., Vandenbusshe, E., \& Vogels, R. (1984). Human orientation discrimination tested with long stimuli. Vision Research, 24, 121-128.

Pelton, L. H., \& Solley, C. M. (1968). Acceleration of reversals of a Necker cube. American Journal of Psychology, 31, 585-589.

Petry, S., \& MEyer, G. E. (1986). Adelphi International Conference on Illusory Contours: A report on the Conference. Perception \& Psychophysics, 39, 210-221.

PraZdNY, K. (1985). On the nature of inducing forms generating perceptions of illusory contours. Perception \& Psychophysics, 37, 237-242.

Pritchard, R. M. (1961). Stabilized images on the retina. Scientific American, 204, 72-78.

Radilova, J., Riani, M., Tuccio, M. T., Radil, T., \& BorselLINO, A. (1983). Reversible figures perceived in two and three dimensional subjective space. Studia Psychologia, 25, 76-79.

Riani, M., Tuocio, M. T., Borseluno, A., Radilova, J., \& Radil, T. (1986). Perceptual ambiguity and stability of reversible figures. Perceptual \& Motor Skills, 63, 191-205.

SChumann, F. (1900). Beiträge zur Analyse der Gesichtswahrnehmungen: Erste Abhandlung. Zeitschrift für Psychologie und Physiologie der Sinnesorgane, 23, 1-32.

VAN Essen, D. C. (1985). Functional organization of primate visual cortex. In A. Peters \& E. G. Jones (Eds.), Cerebral contex: Vol. 3 (pp. 259-329). New York: Plenum.

W ASHBURN, M. F., \& Gillette, A. (1932). Motor factors in voluntary control of cube perspective fluctuations and retinal rivalry fluctuations. American Joumal of Psychology, 44, 315-319.

Wolfe, J. M. (1986). Stereopsis and binocular rivalry. Psychological Review, 93, 269-282.

(Manuscript received December 17, 1990; revision accepted for publication August 12, 1992.) 